\title{
Use of Propofol for Pediatric Percutaneous Renal Needle Biopsy: 164 Cases of Experience
}

\author{
Chen Jie • Lu Kai-zhi
}

Received: 18 May 2011 / Accepted: 30 November 2012 / Published online: 17 January 2013

(C) Dr. K C Chaudhuri Foundation 2013

To the Editor: Over the past decade, propofol has been applied as a rapid and short-acting sedative-hypnotic agent during pediatric invasive procedures [1]. However, there are few studies regarding use of propofol sedation in children undergoing percutaneous renal needle biopsy (PRNB).

We retrospectively analyzed propofol's use in 164 children under the age of $18 \mathrm{y}$ who underwent PRNB between January 2009 and March 2010. Patients were kept fasting for $6 \mathrm{~h}$ before the procedures. Propofol was injected at $1 \mathrm{mg} / \mathrm{s}$ until the desired sedation level was achieved. If necessary, repeated propofol was added as 5-20 $\mathrm{mg}$ bolus every $2 \mathrm{~min}$ to maintain proper sedation to allow the PRNB to continue. All patients underwent standard monitoring and biopsy protocol. Lignocaine skin infiltration was administered before biopsy. Patients were given oxygen via. nasal prongs. After the procedures, patients were watched in the recovery room until recovery of full consciousness.

After analysis of 164 elective pediatric PRNB, a remarkable safety profile of propofol has emerged. Transient hypotension was the most common complication during the procedures. The median decrease in systolic blood pressure was $15 \mathrm{mmHg}$. No vasoactive agents and volume expanders were required to reverse hypotension. Transient desaturation was the second common complication. None of these children needed positive bag ventilation or endotracheal intubation. All of hypotension and desaturation were corrected spontaneously in a short time (less than $5 \mathrm{~min}$ ). There were no episodes of apnea and bradycardia and no PRNB had to be terminated early or aborted for sedation-related issues.
All of the patients were alert within 11 min after the precedures, and the median duration of recovery was $5.4 \mathrm{~min}$.

PRNB is a frightening procedure that is rarely performed in children without sedation [2]. Until recently, most nephrologists use a combination of benzodiazepines and narcotic analgesic agents to induce sedation. However, children usually require more medications and deeper sedation level, often resulting in more side effects. In contrast, propofol has been demonstrated to show rapid onset and termination of action in accompany with better subject comfort and less complications [3]. Current recommendations limit its use in pediatric PRNB, primarily because of the potential for respiratory depression. Our study has shown that propofol has an excellent record of safety and efficacy for sedation in children and patients look very comfortable during the PRNB.

We conclud that propofol is a superior alternative to conventional sedative for invasive procedures.

\section{References}

1. Chen PH, Wu TC, Chiu CY. Pediatric gastrointestinal endoscopic sedation: a 2010 nationwide survey in Taiwan. Pediatr Neonatol. 2012;53:188-92.

2. Blake KD, Madden S, Taylor BW, Rees L. Psychological and clinical effects of renal biopsy performed using sedation. Pediatr Nephrol. 1996;10:693-5.

3. Sebel PS, Lowdon JD. Propofol: a new intravenous anesthetic. Anesthesiology. 1989;71:260-77.
C. Jie $(\bowtie) \cdot$ L. Kai-zhi

Department of Anesthesiology, Southwest Hospital,

Third Military Medical University, 400038, Chongqing, China

e-mail: cjandsh2009@yahoo.cn 